\title{
Use of the SMPTE Test Pattern in Picture Archiving and Communication Systems
}

\author{
Joel E. Gray
}

\begin{abstract}
In 1985 the Society of Motion Picture and Television Engineers (SMPTE) published a Recommended Practice (RP-133) entitled Specifications for Medical Diagnostic Imaging Test Pattern for Television Monitors and Hard-copy Cameras. Since that time the SMPTE monochrome test pattern has been applied to the acceptance testing and quality control of video and image display systems, and hard-copy (film) recorders. The major features of the test pattern will be discussed along with applications and the problems demonstrated with the pattern. Furthermore, the test pattern will be used to demonstrate that color displays may exhibit only one-half of the resolution of a monochrome monitor whereas the display contrast (due to limited dynamic range) may be only 10 to $20 \%$ of that obtained with a monochrome display.

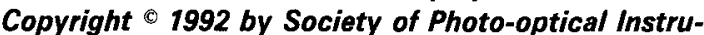
mentation Engineers
\end{abstract}

I N 1981 THE Society of Motion Picture and Television Engineers (SMPTE) formed a committee to develop test procedures for medical image displays and films. The committee consisted of representatives from industry, academic institutions, and clinical radiology departments. Several versions of the pattern were developed and tested, with the final version of the monochrome test pattern being accepted by the SMPTE as a Recommended Practice and published in $1985 .{ }^{1}$

Prior to the development of the test pattern, medical images were evaluated in computer memory of the image acquisition device. Consequently, the output side of the device, the digital-to-analog converter, amplifiers, displays, and hard-copy (film) recorders were not tested. Even if an image of a phantom acquired by the system was displayed and recorded on film, this image could not "stress" the video and display systems or the hard-copy recorders, and it was not possible to separate problems due to the image acquisition, display, and hard-copy record-

From Mayo Clinic, Rochester, $M N$.

Address reprint requests to Joel E. Gray, PhD, Department of Diagnostic Radiology, Mayo Clinic, Rochester, MN 55905.

Copyright $\odot 1992$ by Society of Photo-optical Instrumentation Engineers

0897-1889/92/0501-00/1\$03.00/0 ing portions of the system. Furthermore, no single test pattern was available that could be used for set-up, acceptance testing, and quality control purposes. The goal of the SMPTE committee was to develop such a pattern that could be generated as an analog signal or provided in digital form. In addition, the test pattern was to be useful in both the laboratory and clinical settings, and was to provide both qualitative and quantitative information.

The SMPTE Recommended Practice ${ }^{1}$ describes the attributes and specifications of the test pattern. The SMPTE does not supply the test pattern in digital, analog, or film form. The pattern in digital form can be obtained from most medical imaging equipment vendors. Several video instrumentation firms provide video generators that are capable of producing the SMPTE test pattern in video, analog formatand at virtually any known line rate. Because the test pattern was developed to test medical image displays and hard-copy recorders, and it is extremely difficult to produce a film image with fixed density steps over the range of interest, it is not available as a film image nor have specifications been developed for the pattern in this format. However, it is possible to produce a film image of the SMPTE test pattern using a calibrated hard-copy recorder and then utilize that film to assist in testing film digitizers, video cameras, and other input devices.

The SMPTE test pattern is intended to test medical imaging systems displays and hard-copy recorders for acceptance and quality control purposes in the production facility and the clinical setting. As such, it is a compromise of many needs. Other, more specific patterns are still needed for research purposes and to test specific components in the imaging chain. However, the SMPTE test pattern, as a universally applied pattern, is familiar to many of the medical imaging professionals who are familiar with its features and, more importantly, who know exactly how the image should appear on soft displays and on film. 


\section{DESCRIPTION OF THE TEST PATTERN}

Figure 1 is a schematic drawing of the test pattern. The background of the pattern (1) is a uniform grey at a $50 \%$ video, or average picture, level. This provides the ability to detect uniformity problems and artifacts in the images. Both low and high contrast resolution test patterns ( 3 and 4) are included in the center of the test pattern and in the four corners. The low contrast patterns are all of the same frequency but vary in contrast from $1 \%$ to $5 \%$. The high contrast resolution patterns are at $100 \%$ modulation and vary in frequency with the highest frequency being limited by the pixel size of the digital system, ie, one black pixel followed by one white pixel. A cross-hatch pattern and border (2 and 9) are provided at $75 \%$ of the maximum picture level to assist in determining the effects of spatial distortion and to determine if all of the picture area is displayed or recorded on film.

A grey scale (5) is placed around the center of the image in order to avoid, as much as possible, any falloff in output or light associated with the image display or recording system. The entire dynamic range of the image is represented in 11 steps from 0 to $100 \%$ (in $10 \%$ increments). At either end of the step wedge a smaller $5 \%$ incremental patch (6) is inset in the larger patch. For example, a 5\% patch is inset in the

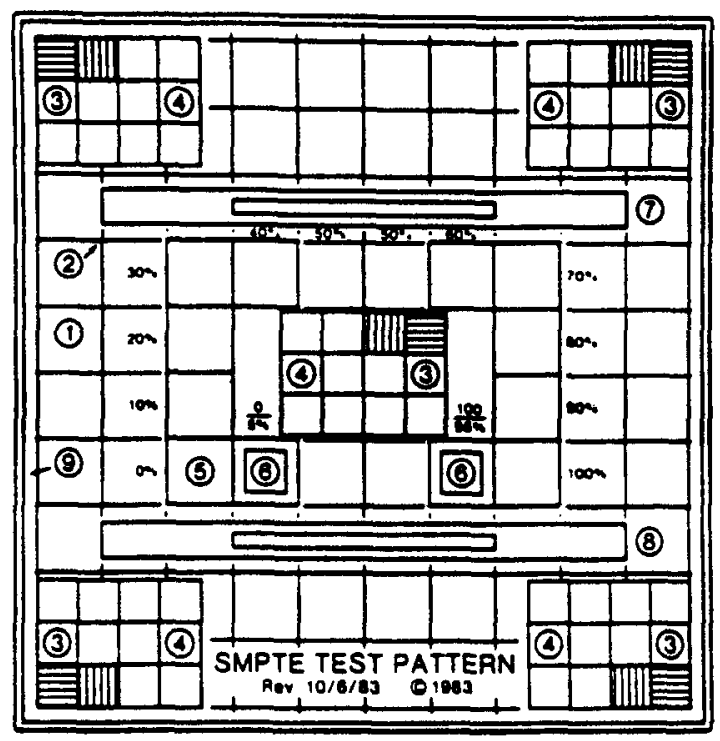

Fig 1. Schematic drawing of SMPTE Test Pattern.
$0 \%$ patch and a $95 \%$ patch is inset in the $100 \%$ patch. These inset patches are useful in deter: mining if the entire range of information available in the test pattern image is being displayed on the image display device or the film. In fact, these patches allow one to easily adjust the contrast and brightness controls of image displays and hard-copy recorders for optimal images in a consistent manner. ${ }^{2}$

Finally, a white and a black window ( 7 and 8 ) are provided to stress the system and test for transient and low frequency response. A well designed imaging system should produce a transition from white to black, or black to white, cleanly, without ringing, overshoot, or smearing.

\section{USE OF THE SMPTE TEST PATTERN}

The test pattern generated by an analog video generator can be used to calibrate the brightness and contrast of video monitors (eg, for fluoroscopy and ultrasound) and video displays (eg, for computed tomography (CT) and magnetic resonance imaging (MRI) systems). This assumes that the video output from the signal generator has the same peak voltage as that from the image source. For this application, the brightness and contrast controls of the monitor are turned completely counter-clockwise (off). The brightness is turned clockwise until a raster pattern is just barely visible in the background. The contrast control is then adjusted until the contrast is maximized and the $95 \%$ patch is visible, without tearing or smearing of the pattern or alphanumerics. (This assumes that the monitor is properly clamped, ie, the black level does not change as the contrast is adjusted. If such changes occur it will be necessary to re-adjust the brightness control so that the background and the raster lines are just barely visible.) As long as the room illumination remains constant these brightness and contrast settings will assure that all of the information in the video signal is displayed on the monitor.

A similar procedure is followed to adjust the brightness and contrast controls for an image display from a digital source. However, one must first assure that the proper window width and level have been selected. The window width must encompass the entire numeric range of the 
test pattern and the level must be set either at the center or appropriate end of that range.

The adjustment of a video or laser hard-copy (film) recorder is also relatively simple. Again, if a digital source is being used it is essential to select the proper window width and level. The controls on the hard-copy recorder are adjusted to produce densities on the film such as those in Table 1. These set-up and quality control levels have been determined based on our experience with over 50 hard-copy recorders (both video and laser) over the past 8 years.

Note that the $0 \%$ patch for the laser hardcopy recorder is set to produce a density of 2.45 even though the film is capable of much higher densities. As indicated earlier, it is the intended that use of the test pattern will help to assure that all information in the data is displayed appropriately. If higher densities are used, the $5 \%$ patch will not be visualized, diagnostic information will be lost on the films, and the films will appear considerably different than the visual display.

It would be possible, in principle, to use a photometer to adjust the brightness and contrast of displays so that one would have exactly the same luminance levels on all displays. In reality this does not work as well as one may anticipate. This is due to the fact that each display may have a slightly different "color" of light output, even though the same phosphor is used for each. This makes quantitative matching of displays difficult. However, it has been our experience and that of others ${ }^{2}$ that it is quite easy to properly adjust a display using the test pattern and the method described previously.

\section{RESULTS WITH MONOCHROME IMAGES}

Results demonstrating the effectiveness of the pattern for monochrome applications have been previously published. ${ }^{3,4}$ Many of the results are associated with video hard-copy recorders including the effect of a raster erase feature, burn of the cathode ray tube (CRT) phosphor, excessive CRT phosphor grain, tearing or smearing of the high density portions of the image, and the effect of two raster erase features (one from the image source and one from the video hard-copy recorder) functioning simultaneously. The test pattern has also been useful in determining the source of problems with laser hardcopy recorders including demonstrating the detrimental effect of some laser hard-copy recorder smoothing algorithms and clearly showing the effect of a laser hard-copy recorder timing problem.

Some imaging systems do not include the capability to correct the image output data to compensate for nonlinearities in the image display or recording systems. A photometer with a fiber optic probe is used to measure the brightness of the test pattern step wedge on the film and visual display. Figure 2 A demonstrates the minimal difference in the luminance from a display and hard-copy image with such correction. Clearly it is possible to produce a film that appears similar to the display with such a system. Figure $2 \mathrm{~B}$ is a similar set of curves for a

Table 1. SMPTE Test Pattern: Set-Up and Quality Control Densities and Density Differences (For CT and MRI)

\begin{tabular}{|c|c|c|c|c|}
\hline \multirow[b]{2}{*}{ SMPTE Patch } & \multicolumn{2}{|c|}{$\begin{array}{l}\text { Video Hard-Copy } \\
\text { Recorder* }\end{array}$} & \multicolumn{2}{|c|}{$\begin{array}{c}\text { Laser Recorder } \\
\text { (Printer)t }\end{array}$} \\
\hline & Recorder Set-Up & Quality Control & Recorder Set-Up & Quality Contro \\
\hline $0 \%$ & $(2.10 \pm 0.08) \ddagger$ & $(2.10 \pm 0.10)$ & $2.45 \pm 0.105$ & $2.45 \pm 0.10$ \\
\hline $10 \%$ & $1.80 \pm 0.10$ & $1.80 \pm 0.10$ & $2.10 \pm 0.10$ & $2.10 \pm 0.10$ \\
\hline $40 \%$ & $1.15 \pm 0.08 \|$ & $1.15 \pm 0.10$ & $1.15 \pm 0.08$ & $1.15 \pm 0.10$ \\
\hline $70 \%$ & $0.50 \pm 0.05$ & & $0.55 \pm 0.05$ & \\
\hline $90 \%$ & $0.28 \pm 0.03$ & $0.28 \pm 0.05$ & $0.30 \pm 0.03$ & $0.30 \pm 0.051$ \\
\hline Density Difference (10\%-70\%) & & $1.30 \pm 0.15$ & & $1.55 \pm 0.15$ \\
\hline
\end{tabular}

*For Kodak NMB film.

tFor Kodak EHN.

\$Maximum density for CT and MRI not controlled for video hard-copy recorders. For ultrasound, nuclear medicine, and digital subtraction angiography for maximum density should be $1.70 \pm 0.10$ (not controlled).

$\S$ Maximum density for laser hard-copy recorders is controlled to prevent it from becoming too dense and loosing information at higher densities.

$\|$ Tighter limits on set-up assures that service engineers set densities closer to operating level.

iDue to slightly higher base-plus-fog of 0.22 . 


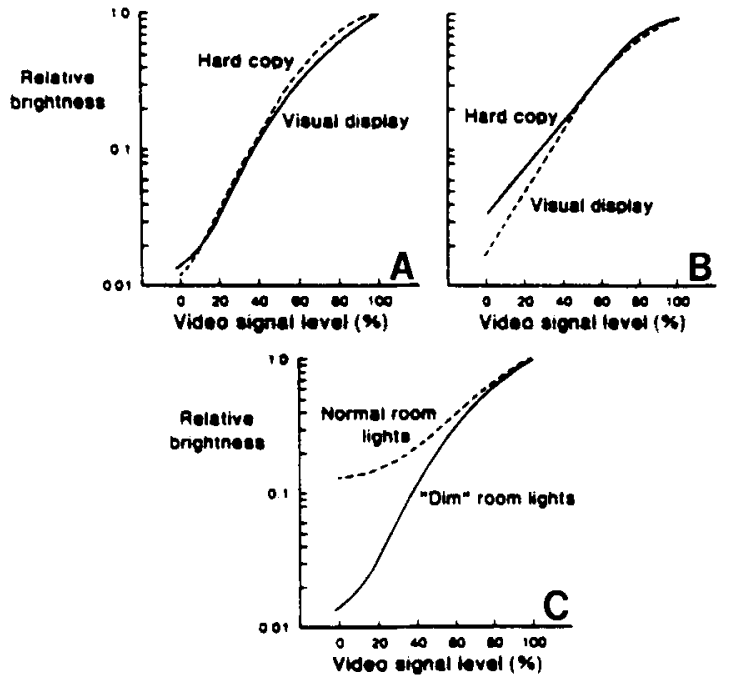

Fig 2. Luminance of visual displays and film images (A) with a look-up table; (B) without look-up table; (C) display only under different lighting conditions.

system that does not includes a look-up table in the image acquisition computer to correct for nonlinearities in the hard-copy recorder. In this case the display image and film image appear significantly different.

The effect of room light on the degradation of dynamic range and image contrast can also be demonstrated with the test pattern (Figure $2 \mathrm{C}$ ). A photometer and fiber optic probe is used to measure the luminance of the display with and without the room lights.

\section{EVALUATION OF COLOR DISPLAYS}

Basic design principles indicate that color displays should exhibit lower resolution and contrast than monochrome displays. Typical monochrome displays have bandwidths of 15
$\mathrm{MHz}$, at a minimum. Conventional color video (TV) displays are typically limited to about 3.5 MHz. Furthermore, the monochrome phosphor is a continuous coating whereas the color phosphor is made up of individual elements of red, green, and blue phosphors, separated by a shadow mask. The "best case" resolution of conventional color displays, be they video or computer driven, is less than one-half of that of monochrome displays. However, some newer, high quality color displays do approach the spatial resolution of monochrome displays. The differences in resolution can be easily demonstrated with the SMPTE test pattern. Other problems are often encountered with color displays including lack of convergence and difficulties in maintaining color purity if the ambient magnetic field exceeds 0.5 gauss, ie, in the vicinity of MRI systems.

The major problem with color displays is the low dynamic range, and hence decreased contrast, when compared to monochrome displays. Each pixel must be made up of three individual phosphor areas, ie, red, green, and blue. This immediately implies that the maximum output of a pixel on a color display is on the order of one-third of the output of the same pixel on the monochrome display. A shadow mask is typically used that reduces the available phosphor area of the color display to about two-thirds that of the monochrome display. These two factors alone result in a "best case" luminance of the color display of approximately $20 \%$ to $25 \%$ of the luminance of the monochrome display. This is clearly demonstrated in Fig 3 . In Fig 3 A the maximum luminance of the color displays is
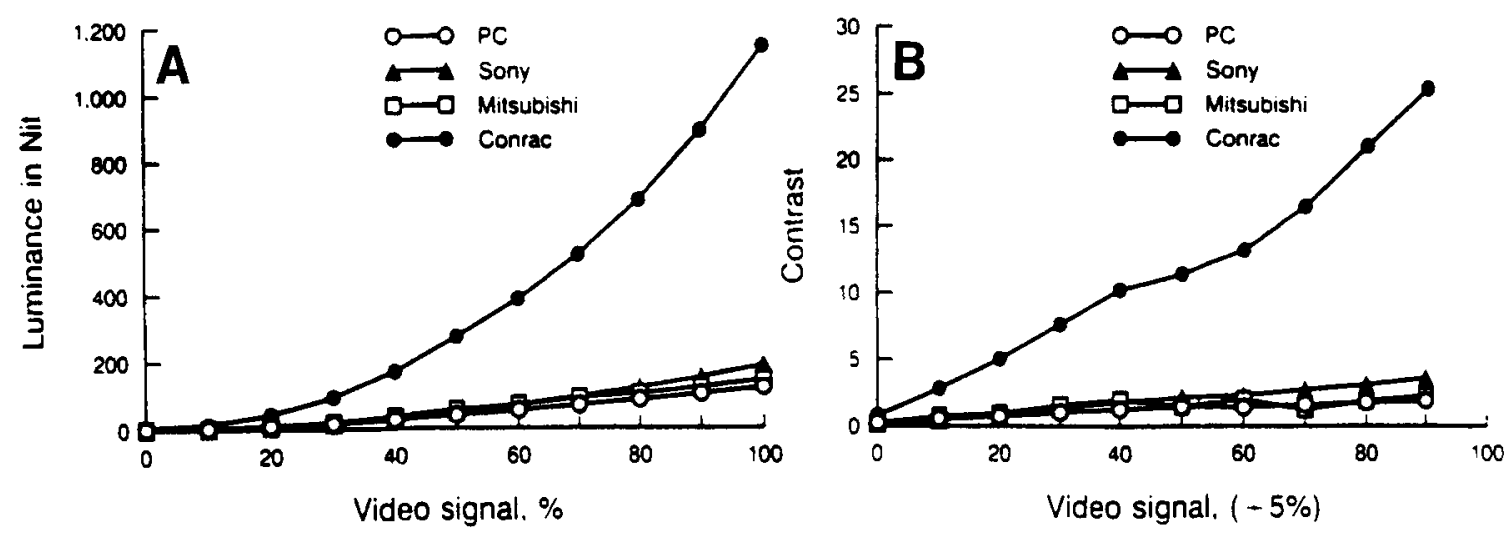

Fig 3. Luminance and contrast of color versus monochrome monitors. 
about $10 \%$ of the luminance of the monochrome display. Likewise, the contrast (luminance per video signal increment) is also decreased proportionately (Fig $3 \mathrm{~B}$ ).

\section{SUMMARY}

The SMPTE test pattern is a useful pattern for evaluating many aspects of both monochrome and color displays, and hard-copy (film) recorders. It provides both quantitative and qualitative information that is useful in both the laboratory and clinical settings. The pattern can be used for evaluation, acceptance testing, and quality control of displays and films. In addition, this pattern has proven beneficial in demonstrating the reduced resolution and contrast, related to limited dynamic range, of color as opposed to monochrome monitors.

\section{REFERENCES}

1. Specifications for medical diagnostic imaging test pattern for television monitors and hard-copy recording cameras. Society of Motion Pictures and Television Engineers (SMPTE) Recommended Practice, RP 133-1986, SMPTE Journal 95:693-695, 1986

2. Bronskill MJ: Experience with the SMPTE test pattern in quality control of magnetic resonance imagers. Proc Society Photo-optical Instrumentation Engineers: Medical Imaging and Instrumentation '84, 486:180-184, 1984
3. Gray JE, Lisk KG, Haddick DH, Members of the SMPTE Subcommittee on Recommended Practices for Medical Diagnostic Display Devices, et al: Test pattern for video displays and hard-copy cameras. Radiology 145:519527,1985

4. Gray JE, Lisk KG, Anderson W, et al: Acceptance and use of the SMPTE medical diagnostic imaging test pattern for television monitors and hard-copy recording cameras. SMPTE Journal 99:1001-1007, 1990 